\title{
Computation of first-order Greeks for barrier options using chain rules for Wiener path integrals
}

\author{
Kensuke Ishitani $^{1}$ \\ 1 Department of Mathematics and Information Sciences, Tokyo Metropolitan University, 1-1 \\ Minami-Osawa, Hachioji, Tokyo 192-0397, Japan \\ E-mailk-ishitani@tmu.ac.jp
}

Received November 14, 2013, Accepted December 19, 2016

\begin{abstract}
This paper presents a new methodology to compute first-order Greeks for barrier options under the framework of path-dependent payoff functions with European, Lookback, or Asian type and with time-dependent trigger levels. In particular, we develop chain rules for Wiener path integrals between two curves that arise in the computation of first-order Greeks for barrier options. We also illustrate the effectiveness of our method through numerical examples.
\end{abstract}

Keywords barrier options, Greeks, 3D Bessel bridge, Brownian meander.

Research Activity Group Mathematical Finance

\section{Introduction and main results}

Barrier options are exotic options whose payoffs depend on whether the underlying asset price reached certain barrier levels (also called trigger levels) prior to maturity. Since barrier options are widely used financial products, computation of their prices and Greeks is an important issue in mathematical finance. Previous works have treated computation of barrier options [1-5] and their Greeks [6] under the framework of specific payoff functions (e.g., European- or Lookback-type functions) with constant trigger levels. This paper proposes a new method for the computation of the Greeks for barrier options under the general framework. Our objective is to compute first-order Greeks for barrier options under the framework of general path-dependent payoff functions with time-dependent option triggers.

The organization of this paper is as follows. This section describes chain rules (CR) for Wiener path integrals between two curves. In Section 2, we formulate the price of double knock-out barrier options under the BlackScholes market model as Wiener path integrals between two curves, and illustrate the effectiveness of our CR method at computing first-order Greeks through European down-and-out call options. Section 3 concludes.

\subsection{Case with two pinned edges}

We borrow some notations from [7]. For $a, b \in \mathbb{R}$ and $T>0$, let $P_{a, b}$ be the (one-dimensional) pinned Wiener measure on $\mathcal{C} \equiv C([0, T])$ such that $w(0)=a$ and $w(T)=b$. We write $w=\{w(t) ; t \in[0, T]\}$ for $w \in \mathcal{C}$. Let two functions $g^{ \pm} \in \mathcal{C}$ be given and assume that $g^{+}$ is strictly above $g^{-}$, namely, they satisfy the condition

$$
g^{-}(t)<g^{+}(t), \quad t \in[0, T] .
$$

We consider

$\mathcal{C}\left\langle g^{-}, g^{+}\right\rangle:=\left\{w \in \mathcal{C} ; g^{-}(t) \leq w(t) \leq g^{+}(t), t \in[0, T]\right\}$, the space of all paths staying between $g^{+}$and $g^{-}$. We first discuss the chain rule for Wiener integrals with re- spect to $P_{a, b}$, restricted on the set $\mathcal{C}\left\langle g^{-}, g^{+}\right\rangle$.

To formulate our main result, we need to introduce several additional notions. For $0 \leq r_{1} \leq r_{2} \leq T$, let $P_{a, b}^{r_{1}, r_{2}}$ be the pinned Wiener measure on $\mathcal{C}^{r_{1}, r_{2}} \equiv$ $C\left(\left[r_{1}, r_{2}\right]\right)$ such that $w\left(r_{1}\right)=a$ and $w\left(r_{2}\right)=b$. We write $\mathcal{C}_{a, b}^{r_{1}, r_{2}}=\left\{w \in \mathcal{C}^{r_{1}, r_{2}} ; w\left(r_{1}\right)=a, w\left(r_{2}\right)=b\right\}$ to indicate the boundary conditions $a$ and $b$.

For a function $g \in \mathcal{C}^{r_{1}, r_{2}}$, we consider $\mathcal{C}_{+}^{r_{1}, r_{2}}\langle g\rangle=$ $\left\{w \in \mathcal{C}^{r_{1}, r_{2}} ; w(t) \leq g(t), t \in\left[r_{1}, r_{2}\right]\right\}$ and $\mathcal{C}_{-}^{r_{1}, r_{2}}\langle g\rangle$, similarly defined by replacing the condition $w(t) \leq$ $g(t)$ with $w(t) \geq g(t)$. As well, $\mathcal{C}_{+}^{r_{1}, r_{2}}\langle g\rangle$ and $\mathcal{C}_{-}^{r_{1}, r_{2}}\langle g\rangle$ are the spaces of all paths staying, respectively, below or above the curve $g$ on the interval $\left[r_{1}, r_{2}\right]$. We set $A_{+}^{r_{1}, r_{2}}(g)=\left(-\infty, g\left(r_{1}\right)\right) \times\left(-\infty, g\left(r_{2}\right)\right)$ and $A_{-}^{r_{1}, r_{2}}(g)=$ $\left(g\left(r_{1}\right), \infty\right) \times\left(g\left(r_{2}\right), \infty\right)$. These are subsets of $\mathbb{R}^{2}$ and specify the classes of boundary conditions $(a, b)$ for the pinned Wiener measures.

If two functions $g^{ \pm} \in \mathcal{C}^{r_{1}, r_{2}}$ satisfying (1) for $t \in\left[r_{1}, r_{2}\right]$ are given, then we set $\mathcal{C}^{r_{1}, r_{2}}\left\langle g^{-}, g^{+}\right\rangle=$ $\mathcal{C}_{+}^{r_{1}, r_{2}}\left\langle g^{+}\right\rangle \cap \mathcal{C}_{-}^{r_{1}, r_{2}}\left\langle g^{-}\right\rangle$and $A^{r_{1}, r_{2}}\left(g^{-}, g^{+}\right)=A_{+}^{r_{1}, r_{2}}\left(g^{+}\right) \cap$ $A_{-}^{r_{1}, r_{2}}\left(g^{-}\right)$. In particular, when $r_{1}=0$ and $r_{2}=T$, we omit the superscripts and write $\mathcal{C}_{a, b}, \quad \mathcal{C}\left\langle g^{-}, g^{+}\right\rangle, \quad A\left(g^{-}, g^{+}\right) \quad$ for $\quad \mathcal{C}_{a, b}^{0, T}, \quad \mathcal{C}^{0, T}\left\langle g^{-}, g^{+}\right\rangle$, $A^{0, T}\left(g^{-}, g^{+}\right)$, and so on. The conditional probabilities $P_{a, b ; g^{-}, g^{+}}^{r_{1}, r_{2}}(\cdot)=P_{a, b}^{r_{1}, r_{2}}\left(\cdot \mid \mathcal{C}^{r_{1}, r_{2}}\left\langle g^{-}, g^{+}\right\rangle\right)$and $P_{a, b ; g, \pm}^{r_{1}, r_{2}}(\cdot)=P_{a, b}^{r_{1}, r_{2}}\left(\cdot \mid \mathcal{C}_{ \pm}^{r_{1}, r_{2}}\langle g\rangle\right)$ of $P_{a, b}^{r_{1}, r_{2}}$ on each path space are defined for $(a, b) \in A^{r_{1}, r_{2}}\left(g^{-}, g^{+}\right)$for $(a, b) \in$ $A_{ \pm}^{r_{1}, r_{2}}(g)$, respectively, in the usual way, and then these definitions can be naturally extended to $(a, b)$ at the boundaries of these sets (see [7, Section 2]).

For $0 \leq r_{1}<r_{2} \leq T$ and $g \in W^{1,2+}\left(\left[r_{1}, r_{2}\right]\right)$, we set

$$
\bar{c}_{\alpha, \beta ; \pm}^{r_{1}, r_{2}}(g)=\frac{2|\alpha-\beta|}{r_{2}-r_{1}} \frac{E^{P_{\alpha, \beta}^{r_{1}, r_{2} ;+}}\left[M_{r_{1}, r_{2}}( \pm g)\right]}{E^{P_{\alpha, \beta}^{r_{1}, r_{2}}}\left[M_{r_{1}, r_{2}}( \pm g)\right]}
$$

for $\alpha, \beta \geq 0$, where $W^{1,2+}\left(\left[r_{1}, r_{2}\right]\right)=\bigcup_{p>2} W^{1, p}\left(\left[r_{1}, r_{2}\right]\right)$ with the usual Sobolev spaces $W^{1, p}\left(\left[r_{1}, r_{2}\right]\right)$ on $\left[r_{1}, r_{2}\right]$, and $P_{\alpha, \beta}^{r_{1}, r_{2} ;+} \equiv P_{\alpha, \beta ; 0,-}^{r_{1}, r_{2}}$ stands for the distribution of the 
three-dimensional Bessel bridge $\left\{w(t) \geq 0 ; t \in\left[r_{1}, r_{2}\right]\right\}$ such that $w\left(r_{1}\right)=\alpha$ and $w\left(r_{2}\right)=\beta$ for $\alpha, \beta \geq 0$ (see [7, Proposition 3.1]). The random variables $M_{r_{1}, r_{2}}(f)$ are Cameron-Martin densities determined by

$$
M_{r_{1}, r_{2}}(g)=\exp \left(\int_{r_{1}}^{r_{2}} g^{\prime}(t) d w(t)-\frac{1}{2} \int_{r_{1}}^{r_{2}} g^{\prime}(t)^{2} d t\right) .
$$

Note that the integrals $\int_{r_{1}}^{r_{2}} g^{\prime}(t) d w(t)$ with $g^{\prime} \in$ $L^{2+}\left(\left[r_{1}, r_{2}\right]\right)=\bigcup_{p>2} L^{p}\left(\left[r_{1}, r_{2}\right]\right)$ are defined as the stochastic integrals (of Wiener type) relative to $w(t)$. See [7, Section 8], particularly for those under $P_{\alpha, \beta}^{r_{1}, r_{2} ;+}$ (with $\alpha$ or $\beta=0$ ).

We further set, for $0<r<T$ and $c \in \mathbb{R}$,

$$
\begin{aligned}
p(r, a, b ; c)= & \frac{p(r, a, c) p(T-r, c, b)}{p(T, a, b)} \\
= & \frac{\sqrt{T}}{\sqrt{2 \pi r(T-r)}} \\
& \quad \times \exp \left(\frac{(a-b)^{2}}{2 T}-\frac{(a-c)^{2}}{2 r}-\frac{(c-b)^{2}}{2(T-r)}\right),
\end{aligned}
$$

where $p(r, a, c)=e^{-(a-c)^{2} / 2 r} / \sqrt{2 \pi r}$.

We are now able to state the first main result of this paper under basic assumptions on the curves $g_{[\lambda]}^{ \pm}$: $[0, T] \rightarrow \mathbb{R}$ and the functionals $F_{[\lambda]}: \mathcal{C}_{a, b} \rightarrow \mathbb{R}(\lambda \in \Lambda)$, where $\Lambda$ is an open subset of $\mathbb{R}$. The following are the conditions for $\left\{g_{[\lambda]}^{ \pm}\right\}_{\lambda \in \Lambda}=\left\{g_{[\lambda]}^{-}, g_{[\lambda]}^{+}\right\}_{\lambda \in \Lambda}$ :

[g1] For $\lambda \in \Lambda$, two curves $g_{[\lambda]}^{ \pm}=\left\{g_{[\lambda]}^{ \pm}(t)\right\}_{t \in[0, T]}$ belong to $W^{1,2+}([0, T])$, and satisfy $(1)$.

$[\mathrm{g} 2]$ For $t \in[0, T], g_{[\cdot]}^{ \pm}(t):=\left\{g_{[\lambda]}^{ \pm}(t)\right\}_{\lambda \in \Lambda}$ belong to $\mathcal{C}^{1}(\Lambda)$. In addition, for $\lambda \in \Lambda$, $\partial g_{[\lambda]}^{ \pm} / \partial \lambda:=\left\{\partial g_{[\lambda]}^{ \pm}(t) / \partial \lambda\right\}_{t \in[0, T]}$ belong to the class $W^{1,2+}([0, T])$.

The following are the conditions for $\left\{F_{[\lambda]}\right\}_{\lambda \in \Lambda}$ :

[F1] For $w \in \mathcal{C}_{a, b}, \quad F_{[\cdot]}(w):=\left\{F_{[\lambda]}(w)\right\}_{\lambda \in \Lambda}$ is a function from $\Lambda$ to $\mathbb{R}$, and belongs to $\mathcal{C}^{1}(\Lambda)$. We denote the derivative of $F_{[\lambda]}(w)$ with respect to $\lambda$ by $\partial F_{[\lambda]}(w) / \partial \lambda$, and write $\partial F_{[\lambda]}(\cdot) / \partial \lambda=$ $\left\{\partial F_{[\lambda]}(w) / \partial \lambda\right\}_{w \in \mathcal{C}_{a, b}}$.

[F2] For $\lambda \in \Lambda, F_{[\lambda]}(\cdot)$ and $\partial F_{[\lambda]}(\cdot) / \partial \lambda$ are bounded continuous functionals on $\mathcal{C}_{a, b} \cap \mathcal{C}\left\langle g_{[\lambda]}^{-}, g_{[\lambda]}^{+}\right\rangle$.

For $0 \leq r_{1}<r_{2} \leq T$ and $g \in \mathcal{C}$, the expression $\left.g\right|_{\left[r_{1}, r_{2}\right]} \in$ $\mathcal{C}^{r_{1}, r_{2}}$ gives the restriction of $g$ on interval $\left[r_{1}, r_{2}\right]$.

Theorem 1 Assume that $\widetilde{A}:=\cap_{\lambda \in \Lambda} A\left(g_{[\lambda]}^{-}, g_{[\lambda]}^{+}\right) \neq \emptyset$ and $(a, b) \in \widetilde{A}$. Then, the Wiener path integral

$$
\Phi_{a, b}(\lambda):=\int_{\mathcal{C}\left\langle g_{[\lambda]}^{-}, g_{[\lambda]}^{+}\right\rangle} F_{[\lambda]}(w) P_{a, b}(d w) \quad(\lambda \in \Lambda)
$$

is differentiable with respect to $\lambda \in \Lambda$, and we have

$$
\begin{aligned}
& \qquad \begin{aligned}
& \frac{\partial}{\partial \lambda} \Phi_{a, b}(\lambda)=I_{a, b}(\lambda)+B_{a, b}^{+}(\lambda)-B_{a, b}^{-}(\lambda) \quad(\lambda \in \Lambda), \\
& \text { where } \\
& I_{a, b}(\lambda)=\int_{\mathcal{C}\left\langle g_{[\lambda]}^{-}, g_{[\lambda]}^{+}\right\rangle} \frac{\partial}{\partial \lambda} F_{[\lambda]}(w) P_{a, b}(d w), \\
& B_{a, b}^{ \pm}(\lambda)=\int_{0}^{T} \frac{\partial}{\partial \lambda} g_{[\lambda]}^{ \pm}(r) \nu_{a, b}^{ \pm}(r) \\
& E_{a, b}^{r, \pm}\left[F_{[\lambda]}\left(\left.w\right|_{[0, r]},\left.w\right|_{[r, T]}\right)\right] d r
\end{aligned}
\end{aligned}
$$

and the expectations $E_{a, b}^{r, \pm}$ in the right-hand side are found under the product of the conditional probabilities

$$
P_{a, g_{[\lambda]}^{ \pm}(r) ; g_{[\lambda]}^{-}, g_{[\lambda]}^{+}}^{0, r} \otimes P_{g_{[\lambda]}^{ \pm}}^{r, T}(r), b ; g_{[\lambda]}^{-}, g_{[\lambda]}^{+}
$$

for $w=\left(\left.w\right|_{[0, r]},\left.w\right|_{[r, T]}\right)$, respectively, and for $r \in(0, T)$

$$
\begin{aligned}
\nu_{a, b}^{ \pm}(r) & \\
:= & \frac{1}{2} p\left(r, a, b ; g_{[\lambda]}^{ \pm}(r)\right) \\
& \times \bar{c}_{\left|a-g_{[\lambda]}^{ \pm}(0)\right|, 0 ; \pm}^{0, r}\left(g_{[\lambda]}^{ \pm}\right) P_{a, g_{[\lambda]}^{ \pm}(r) ; g_{[\lambda]}^{ \pm}, \pm}^{0, r}\left(\mathcal{C}_{\mp}^{0, r}\left\langle g_{[\lambda]}^{\mp}\right\rangle\right) \\
& \times \bar{c}_{0,\left|b-g_{[\lambda]}^{ \pm}(T)\right| ; \pm}^{r, T}\left(g_{[\lambda]}^{ \pm}\right) P_{g_{[\lambda]}^{ \pm}(r), b ; g_{[\lambda]}^{ \pm}, \pm}^{ \pm}\left(\mathcal{C}_{\mp}^{r, T}\left\langle g_{[\lambda]}^{\mp}\right\rangle\right) .
\end{aligned}
$$

\subsection{Case with one pinned edge and one free edge}

We next consider the case where $w(T)$ can move freely. For $0 \leq r_{1}<r_{2} \leq T$, let $P_{a}^{r_{1}, r_{2}}$ be the Wiener measure on $\mathcal{C}_{a}^{r_{1}, r_{2}}:=\left\{w \in \mathcal{C}^{r_{1}, r_{2}} ; w\left(r_{1}\right)=a\right\}$. For $0 \leq r \leq T$ and $g, g^{ \pm} \in \mathcal{C}^{r, T}$, we set $B_{+}^{r}(g)=(-\infty, g(r))$, $B_{-}^{r}(g)=(g(r), \infty)$ and $B^{r}\left(g^{-}, g^{+}\right)=\left(g^{-}(r), g^{+}(r)\right)$. These subsets of $\mathbb{R}$ specify the classes of boundary conditions $a$ for $P_{a}^{r_{1}, r_{2}}$ at $r=r_{1}$. When $r_{1}=0, r_{2}=T$ or $r=0$, we drop the superscripts and write $P_{a}, \mathcal{C}_{a}$, $B\left(g^{-}, g^{+}\right)$for $P_{a}^{0, T}, \mathcal{C}_{a}^{0, T}, B^{0}\left(g^{-}, g^{+}\right)$, and so on. In this subsection, we discuss the chain rule for integrals with respect to $P_{a}$ restricted on the set $\mathcal{C}\left\langle g^{-}, g^{+}\right\rangle$. For $g \in \mathcal{C}^{r_{1}, r_{2}}$ and $g^{ \pm} \in \mathcal{C}^{r_{1}, r_{2}}$ satisfying (1), the conditional probabilities $P_{a ; g^{-}, g^{+}}^{r_{1}, r_{2}}(\cdot)=P_{a}^{r_{1}, r_{2}}\left(\cdot \mid \mathcal{C}^{r_{1}, r_{2}}\left\langle g^{-}, g^{+}\right\rangle\right)$and $P_{a ; g, \pm}^{r_{1}, r_{2}}(\cdot)=P_{a}^{r_{1}, r_{2}}\left(\cdot \mid \mathcal{C}_{ \pm}^{r_{1}, r_{2}}\langle g\rangle\right)$ of $P_{a}^{r_{1}, r_{2}}$ are defined for $a \in B^{r_{1}}\left(g^{-}, g^{+}\right)$for $a \in B_{ \pm}^{r_{1}}(g)$, respectively, in the usual way, and again these definitions can be extended to $a$ at the boundaries of these sets (see [7, Section 5]). For $0 \leq r_{1}<r_{2} \leq T$ and $g \in W^{1,2+}\left(\left[r_{1}, r_{2}\right]\right)$, we set

$$
\bar{d}_{ \pm}^{r_{1}, r_{2}}(g)=\sqrt{\frac{2}{\pi\left(r_{2}-r_{1}\right)}} E^{P_{0}^{r_{1}, r_{2} ;+}}\left[M_{r_{1}, r_{2}}( \pm g)\right],
$$

where $P_{\alpha}^{r_{1}, r_{2} ;+}:=P_{\alpha ; 0,-}^{r_{1}, r_{2}}$ stands for the distribution of the Brownian meander $\left\{w(t) \geq 0 ; t \in\left[r_{1}, r_{2}\right]\right\}$ such that $w\left(r_{1}\right)=\alpha \geq 0$. See Proposition 5.4 for $\bar{d}_{-}^{0, r}(g)$ and Section 8 in [7] for the stochastic integrals.

We now state the second main result of this paper. We assume that the curves $\left\{g_{[\lambda]}^{ \pm}\right\}_{\lambda \in \Lambda}=\left\{g_{[\lambda]}^{-}, g_{[\lambda]}^{+}\right\}_{\lambda \in \Lambda}$ satisfy the conditions [g1] and [g2]. The following are the condition for the functionals $\left\{F_{[\lambda]}\right\}_{\lambda \in \Lambda}$ :

[F3] For $\lambda \in \Lambda$, the conditions [F1] and [F2] are satisfied for almost every $b \in B\left(g_{[\lambda]}^{-}, g_{[\lambda]}^{+}\right)$. It holds that

$$
\widehat{M}_{a}(\lambda):=\operatorname{esssup}_{b \in B\left(g_{[\lambda]}^{-}, g_{[\lambda]}^{+}\right)} \widehat{M}_{a, b}(\lambda)<\infty \quad(\lambda \in \Lambda),
$$
where

$$
\widehat{M}_{a, b}(\lambda):=\sup _{w \in \mathcal{C}_{a, b} \cap \mathcal{C}\left\langle g_{[\lambda]}^{-}, g_{[\lambda]}^{+}\right\rangle}\left(\left|F_{[\lambda]}(w)\right| \vee\left|\frac{\partial}{\partial \lambda} F_{[\lambda]}(w)\right|\right) .
$$

Theorem 2 Assume that $\widetilde{B}:=\cap_{\lambda \in \Lambda} B\left(g_{[\lambda]}^{-}, g_{[\lambda]}^{+}\right) \neq \emptyset$ and $a \in \widetilde{B}$. Then,

$$
\Phi_{a}(\lambda):=\int_{\mathcal{C}\left\langle g_{[\lambda]}^{-}, g_{[\lambda]}^{+}\right\rangle} F_{[\lambda]}(w) P_{a}(d w) \quad(\lambda \in \Lambda)
$$

is differentiable with respect to $\lambda \in \Lambda$, and we have

$$
\frac{\partial}{\partial \lambda} \Phi_{a}(\lambda)=I_{a}(\lambda)+B_{a}^{+}(\lambda)-B_{a}^{-}(\lambda) \quad(\lambda \in \Lambda),
$$


where

$I_{a}(\lambda)=\int_{\mathcal{C}\left\langle g_{[\lambda]}^{-}, g_{[\lambda]}^{+}\right\rangle} \frac{\partial}{\partial \lambda} F_{[\lambda]}(w) P_{a}(d w)$,

$B_{a}^{ \pm}(\lambda)=\int_{0}^{T} \frac{\partial}{\partial \lambda} g_{[\lambda]}^{ \pm}(r) \nu_{a}^{ \pm}(r) E_{a}^{r, \pm}\left[F_{[\lambda]}\left(\left.w\right|_{[0, r]},\left.w\right|_{[r, T]}\right)\right] d r$

and the expectations $E_{a}^{r, \pm}$ in the right-hand side are found under the product of the conditional probabilities

$$
P_{a, g_{[\lambda]}^{ \pm}(r) ; g_{[\lambda]}^{-}, g_{[\lambda]}^{+}}^{0, r} \otimes P_{g_{[\lambda]}^{ \pm}}^{r, T}(r) ; g_{[\lambda]}^{-}, g_{[\lambda]}^{+}
$$

for $w=\left(\left.w\right|_{[0, r]},\left.w\right|_{[r, T]}\right)$, respectively, and for $r \in(0, T)$

$$
\begin{aligned}
& \nu_{a}^{ \pm}(r):=\frac{1}{2} p\left(r, a, g_{[\lambda]}^{ \pm}(r)\right) \\
& \times \bar{c}_{\left|a-g_{[\lambda]}^{ \pm}(0)\right|, 0 ; \pm}^{ \pm, r}\left(g_{[\lambda]}^{ \pm}\right) P_{a, g_{[\lambda]}^{ \pm}(r) ; g_{[\lambda]}^{ \pm}, \pm}^{0, r}\left(\mathcal{C}_{\mp}^{0, r}\left\langle g_{[\lambda]}^{\mp}\right\rangle\right) \\
& \times \bar{d}_{ \pm}^{r, T}\left(g_{[\lambda]}^{ \pm}\right) P_{g_{[\lambda]}^{ \pm}(r) ; g_{[\lambda]}^{ \pm}, \pm}^{r, T}\left(\mathcal{C}_{\mp}^{r, T}\left\langle g_{[\lambda]}^{\mp}\right\rangle\right) .
\end{aligned}
$$

\subsection{Related results}

Integration by parts formulas (IbPFs) for Wiener measures on a path space between two curves are established in [7]. As well, Theorems 1 and 2 give the CRs for Wiener path integrals between two curves. The approaches for both proofs are based on the classical polygonal approximations for the Brownian motions, which reduce the IbPFs and the CRs to those on finite-dimensional spaces. Note that $B_{a, b}^{ \pm}(\lambda)$ and $B_{a}^{ \pm}(\lambda)$ are quite similar to the boundary term of IbPFs in [7].

\section{Application of CRs to compute first- order Greeks for barrier options}

We formulate the price of double knock-out barrier options under the Black-Scholes market model as Wiener path integrals between two curves. The security price process $S=\left\{S_{t}\right\}_{0 \leq t \leq T}$ is assumed to follow a stochastic differential equation of the form

$$
d S_{t}=\mu S_{t} d t+\sigma S_{t} d w(t),
$$

where $T, S_{0}, \sigma>0, \mu \in \mathbb{R}$, and where $w=\{w(t)\}_{t \in[0, T]}$ is the standard one-dimensional Brownian motion defined on the probability space $\left(\mathcal{C}^{0, T}, \mathcal{B}\left(\mathcal{C}^{0, T}\right), P\right)$. Let us note that the value of a knock-out barrier option can be expressed as the following form:

$$
\widehat{\Phi}:=E\left[e^{-c T} f(S) 1_{\mathcal{C}\left\langle e^{G^{-}}, e^{G^{+}}\right\rangle}(S)\right] .
$$

Here, $T$ is a maturity of the option, $f: \mathcal{C}^{0, T} \rightarrow \mathbb{R}$ is an option payoff function, and $c \geq 0$ stands for the risk-free interest rate. Moreover, $G^{ \pm}=\left\{G^{ \pm}(t)\right\}_{t \in[0, T]} \in$ $W^{1,2+}([0, T])$ satisfying (1) stand for trigger curves for the logarithmic process $X=\left\{X_{t}:=\log S_{t}\right\}_{0 \leq t \leq T}$. We define $g^{ \pm}=\left\{g^{ \pm}(t)\right\}_{t \in[0, T]}$ and $F(\cdot)=\{F(w)\}_{w \in \mathcal{C}_{0}}$ by

$$
\begin{aligned}
& g^{ \pm}(t):=\frac{1}{\sigma}\left[G^{ \pm}(t)-\log S_{0}-\left(\mu-\frac{\sigma^{2}}{2}\right) t\right], \\
& F(w):=e^{-c T} f\left(\left\{e^{\left(\mu-\sigma^{2} / 2\right) t+\sigma w(t)}\right\}_{0 \leq t \leq T}\right) .
\end{aligned}
$$

Note that $g^{ \pm}$are the triggers for $w=\{w(t)\}_{t \in[0, T]}$. Thus, it holds that $1_{\mathcal{C}\left\langle G^{-}, G^{+}\right\rangle}(X)=1_{\mathcal{C}\left\langle g^{-}, g^{+}\right\rangle}(w)$ and

$$
\widehat{\Phi}=\int_{\mathcal{C}\left\langle g^{-}, g^{+}\right\rangle} F(w) P_{0}(d w) .
$$

Note that $\partial \widehat{\Phi} / \partial \sigma$ and $\partial \widehat{\Phi} / \partial S_{0}$ are Vega and Delta, respectively, for this barrier option. Since $\partial g^{ \pm}(t) / \partial \sigma=$ $\left[-G^{ \pm}(t)+\log S_{0}+\left(\mu+\sigma^{2} / 2\right) t\right] / \sigma^{2}, \partial g^{ \pm}(t) / \partial S_{0}=$ $-1 /\left(\sigma S_{0}\right)$ hold for $t \in[0, T]$, both $[g 1]$ and $[g 2]$ are satisfied for $g^{ \pm}$with $\lambda=\sigma$ or $\lambda=S_{0}$.

\subsection{European down-and-out call option}

This subsection demonstrates the effectiveness of our CR to compute first-order Greeks by using European down-and-out call options whose Greeks can also be calculated directly. The characteristic of this type of option is that if the security price $S=\left\{S_{t}\right\}_{0 \leq t \leq T}$ ever reaches the lower trigger level $e^{G^{-}(t)} \equiv L \in\left(0, S_{0}\right)$, then the right of the European call option is extinguished. The price of this option is given as the following expectation:

$$
C_{\text {Barrier }}^{\mathrm{BS}}\left(S_{0}\right):=\int_{\mathcal{C}_{-}^{0, T}\left\langle g^{-}\right\rangle} F(w) P_{0}(d w),
$$

where the payoff function $F(w)$ is given as

$$
F(w)=e^{-c T}\left(S_{0} e^{\left(\mu-\sigma^{2} / 2\right) T+\sigma w(T)}-K\right)^{+}, \quad w \in \mathcal{C}_{0},
$$

with a strike price $K(\geq L)$. Using the joint distribution of $w(T)$ and $\min _{t \in[0, T]} w(t)$, the price of a European down-and-out call option can be calculated as follows:

$$
C_{\text {Barrier }}^{\mathrm{BS}}\left(S_{0}\right)=C^{\mathrm{BS}}\left(S_{0}\right)-\left(\frac{S_{0}}{L}\right)^{1-\frac{2 \mu}{\sigma^{2}}} C^{\mathrm{BS}}\left(\frac{L^{2}}{S_{0}}\right),
$$

where $C^{\mathrm{BS}}\left(S_{0}\right):=E\left[e^{-c T}\left(S_{T}-K\right)^{+}\right]$is the price of a European call option (vanilla option). Note also that the price of a European call option can be calculated as $C^{\mathrm{BS}}\left(S_{0}\right)=e^{-c T}\left[e^{\mu T} S_{0} \mathcal{N}\left(d_{1}\left(T, X_{0}\right)\right)-K \mathcal{N}\left(d_{2}\left(T, X_{0}\right)\right)\right]$, where $\mathcal{N}(x):=\int_{-\infty}^{x} n(z) d z, n(z):=e^{-z^{2} / 2} / \sqrt{2 \pi}$,

$$
\begin{aligned}
& d_{1}(t, x):=\frac{1}{\sigma \sqrt{t}}\left[x-\log K+\left(\mu+\frac{\sigma^{2}}{2}\right) t\right], \\
& d_{2}(t, x):=d_{1}(t, x)-\sigma \sqrt{t} \quad(t>0, x \in \mathbb{R}) .
\end{aligned}
$$

Using (7), Vega and Delta of the European down-andout call option can be calculated directly as follows: Vega(Direct)

$$
\begin{aligned}
= & \frac{\partial}{\partial \sigma} C_{\text {Barrier }}^{\mathrm{BS}}\left(S_{0}\right) \\
= & \frac{e^{-c T}}{\sigma}\left(K d_{1} n\left(d_{2}\right)-e^{\mu T} S_{0} d_{2} n\left(d_{1}\right)\right) \\
& +\left(\frac{S_{0}}{L}\right)^{1-2 \mu / \sigma^{2}} \frac{e^{(\mu-c) T}}{\sigma}\left(\frac{L^{2}}{S_{0}}\right) \widetilde{d}_{2} n\left(\widetilde{d}_{1}\right) \\
& -\left(\frac{S_{0}}{L}\right)^{1-2 \mu / \sigma^{2}} \frac{e^{-c T}}{\sigma} K \widetilde{d}_{1} n\left(\widetilde{d}_{2}\right) \\
& +4\left(\frac{\mu}{\sigma^{3}}\right)\left(\frac{S_{0}}{L}\right)^{1-2 \mu / \sigma^{2}} \log \left(\frac{S_{0}}{L}\right) e^{-c T} \\
& \times\left[K \mathcal{N}\left(\widetilde{d}_{2}\right)-e^{\mu T}\left(\frac{L^{2}}{S_{0}}\right) \mathcal{N}\left(\widetilde{d}_{1}\right)\right],
\end{aligned}
$$

$\operatorname{Delta}($ Direct)

$$
\begin{aligned}
& =\frac{\partial}{\partial S_{0}} C_{\text {Barrier }}^{\mathrm{BS}}\left(S_{0}\right) \\
& =e^{(\mu-c) T} \mathcal{N}\left(d_{1}\right)+e^{(\mu-c) T} \frac{n\left(d_{1}\right)}{\sigma \sqrt{T}}-e^{-c T} K \frac{n\left(d_{2}\right)}{S_{0} \sigma \sqrt{T}}
\end{aligned}
$$


Table 1. The results of Vega for $\mu= \pm 0.1, \pm 0.3$.

\begin{tabular}{|c|c||c|cc|}
\hline \hline \multicolumn{5}{|c|}{ Vega } \\
\hline$\mu$ & Direct & CR & $I_{0}(\sigma)$ & $B_{0}^{-}(\sigma)$ \\
\hline \hline-0.3 & 2.02572 & 2.02592 & 2.03173 & 0.00580274 \\
-0.1 & 11.8716 & 11.8716 & 14.2908 & 2.41917 \\
0.1 & 7.2316 & 7.2316 & 29.7194 & 22.4877 \\
0.3 & -44.3372 & -44.3372 & 22.4359 & 66.7731 \\
\hline
\end{tabular}

Table 2. The results of Delta for $\mu= \pm 0.1, \pm 0.3$.

\begin{tabular}{|c|c||c|cc|}
\hline \hline \multicolumn{5}{|c|}{ Delta } \\
\hline$\mu$ & Direct & CR & $I_{0}\left(S_{0}\right)$ & $B_{0}^{-}\left(S_{0}\right)$ \\
\hline \hline-0.3 & 0.016236 & 0.0162332 & 0.00952157 & -0.0067116 \\
-0.1 & 0.205788 & 0.205789 & 0.108803 & -0.096986 \\
0.1 & 0.966049 & 0.966049 & 0.457254 & -0.508795 \\
0.3 & 2.10324 & 2.10324 & 0.932094 & -1.17115 \\
\hline
\end{tabular}

$$
\begin{aligned}
& -\left(\frac{S_{0}}{L}\right)^{1-2 \mu / \sigma^{2}} \frac{\left(1-2 \frac{\mu}{\sigma^{2}}\right) C^{\mathrm{BS}}\left(\frac{L^{2}}{S_{0}}\right)}{S_{0}} \\
& +\left(\frac{S_{0}}{L}\right)^{1-2 \mu / \sigma^{2}} \frac{e^{(\mu-c) T}\left(\frac{L^{2}}{S_{0}}\right) n\left(\widetilde{d}_{1}\right)-e^{-c T} K n\left(\widetilde{d}_{2}\right)}{S_{0} \sigma \sqrt{T}} \\
& +\left(\frac{S_{0}}{L}\right)^{1-2 \mu / \sigma^{2}} e^{(\mu-c) T}\left(\frac{L}{S_{0}}\right)^{2} \mathcal{N}\left(\widetilde{d}_{1}\right)
\end{aligned}
$$

where $\left\{d_{i}, \widetilde{d}_{i}\right\}_{i=1,2}$ are given as $d_{i}:=d_{i}\left(T, \log S_{0}\right), \widetilde{d}_{i}:=$ $d_{i}\left(T, \log \left(L^{2} / S_{0}\right)\right)(i=1,2)$.

In contrast, the CR formula (6) in Theorem 2 presents a new method to compute the Greeks of barrier options with time-dependent trigger levels and path-dependent payoff functions. Thus, it is also meaningful to apply (6) to compute Vega and Delta for a European down-andout call option, which is explained below. The derivatives of the payoff function are given as

$$
\begin{aligned}
& \frac{\partial F}{\partial \sigma}(w)=e^{-c T}(-\sigma T+w(T)) S_{0} e^{\left(\mu-\frac{\sigma^{2}}{2}\right) T+\sigma w(T)}, \\
& \frac{\partial F}{\partial S_{0}}(w)=e^{-c T} e^{\left(\mu-\frac{\sigma^{2}}{2}\right) T+\sigma w(T)}
\end{aligned}
$$

for $w \in \mathcal{C}_{0}$ satisfying $w(T)>-\sqrt{T} d_{2}\left(T, X_{0}\right)$, and $\partial F(w) / \partial \sigma=\partial F(w) / \partial S_{0}=0$ for $w \in \mathcal{C}_{0}$ satisfying $w(T)<-\sqrt{T} d_{2}\left(T, X_{0}\right)$. Thus, [F3] is satisfied for $\lambda=\sigma$ and $\lambda=S_{0}$. Since $g^{+}(t) \equiv \infty$, it holds that $B_{0}^{+}(\sigma)=B_{0}^{+}\left(S_{0}\right)=0$. Therefore, using (6), we have

$$
\begin{array}{ll}
\operatorname{Vega}(\mathrm{CR}):=I_{0}(\sigma)-B_{0}^{-}(\sigma) & (\sigma>0), \\
\operatorname{Delta}(\mathrm{CR}):=I_{0}\left(S_{0}\right)-B_{0}^{-}\left(S_{0}\right) & \left(S_{0}>0\right) .
\end{array}
$$

Using (10), (11), and the joint distribution of $w(T)$ and $\min _{t \in[0, T]} w(t)$, we can compute $I_{0}(\sigma)$ and $I_{0}\left(S_{0}\right)$ as

$$
\begin{aligned}
I_{0}(\sigma)=e^{(\mu-c) T} S_{0} \sqrt{T} n\left(d_{1}\right) & \\
& +\left(\frac{L}{S_{0}}\right)^{1+\frac{2 \mu}{\sigma^{2}}} e^{(\mu-c) T} S_{0} \\
& \times\left[\frac{\log \left(\frac{S_{0}}{L}\right)^{2}}{\sigma} \mathcal{N}\left(\widetilde{d}_{1}\right)-\sqrt{T} n\left(\widetilde{d}_{1}\right)\right], \\
I_{0}\left(S_{0}\right)= & e^{(\mu-c) T}\left[\mathcal{N}\left(d_{1}\right)-\left(\frac{L}{S_{0}}\right)^{1+\frac{2 \mu}{\sigma^{2}}} \mathcal{N}\left(\widetilde{d}_{1}\right)\right] .
\end{aligned}
$$

Additionally, the definition of $B_{0}^{-}(\lambda)$ implies $B_{0}^{-}(\sigma)=\int_{0}^{T} \frac{\partial g^{-}}{\partial \sigma}(r) \nu_{0}^{-}(r) E_{0}^{r,-}\left[F\left(\left.w\right|_{[0, r]},\left.w\right|_{[r, T]}\right)\right] d r$,

$$
B_{0}^{-}\left(S_{0}\right)=\int_{0}^{T} \frac{\partial g^{-}}{\partial S_{0}}(r) \nu_{0}^{-}(r) E_{0}^{r,-}\left[F\left(\left.w\right|_{[0, r]},\left.w\right|_{[r, T]}\right)\right] d r .
$$

Moreover, we can obtain the following equation by using the definition of $\nu_{0}^{-}(r)$ and the distribution of the Brownian meander $\left.w\right|_{[r, T]}=\left\{\left.w\right|_{[r, T]}(t)\right\}_{t \in[r, T]}$ :

$$
\begin{aligned}
\nu_{0}^{-}(r) E_{0}^{r,-}\left[F\left(\left.w\right|_{[0, r]},\left.w\right|_{[r, T]}\right)\right] & \\
= & \log \left(\frac{S_{0}}{L}\right) \frac{2 e^{-c T}}{\sigma r \sqrt{r}} \\
\times & \exp \left(-(T-r) \frac{\kappa_{-}^{2}}{2}\right) n\left(\frac{\log \left(S_{0} / L\right)}{\sigma \sqrt{r}}+\kappa_{-} \sqrt{r}\right) \\
\times & {\left[L \kappa_{+} \exp \left(\frac{(T-r) \kappa_{+}^{2}}{2}\right) \mathcal{N}\left(d_{1}(T-r, \log L)\right)\right.} \\
& \left.\quad-K \kappa_{-} \exp \left(\frac{(T-r) \kappa_{-}^{2}}{2}\right) \mathcal{N}\left(d_{2}(T-r, \log L)\right)\right],
\end{aligned}
$$

where $\kappa_{ \pm}:=\mu / \sigma \pm \sigma / 2$. Note that [5] obtained an approximation formula for the down-and-out barrier European call option under the stochastic volatility model, and their first-order coefficient function is similar to (12).

In the following, we set $c=0, T=0.75, S_{0}=100$, $K=105, L=95$, and $\sigma=0.15$. We examine four patterns for $\mu= \pm 0.1, \pm 0.3$, and partition $[0, T]$ into 100,000 equal intervals to apply the trapezoidal rule to compute the integrals of $B_{0}^{-}(\sigma)$ and $B_{0}^{-}\left(S_{0}\right)$. Table 1 and Table 2 show the results of Vega in (8) and (12), and Delta in (9) and (13), respectively. We can confirm that our CR method can accurately calculate Delta and Vega for the down-and-out barrier European call option.

\section{Conclusion and discussions}

We introduced our newly developed CR method to compute first-order Greeks for barrier options under the framework of path-dependent payoff functions with time-dependent trigger levels. We also demonstrated that our method can accurately compute first-order Greeks, applying it to European down-and-out call options under the Black-Scholes market model. We are currently investigating the $\mathrm{CR}$ formulas for computing higher-order Greeks of barrier options under the general market model. We hope to report more extensive and challenging results in a future article.

\section{References}

[1] R. C. Merton, Theory of rational option pricing, Bell J. Econ., 4 (1973), 141-183.

[2] N. Kunitomo and M. Ikeda, Pricing options with curved boundaries, Math. Finan., 2 (1992), 275-298.

[3] Y. Muroi, Pricing lookback options with knock-out boundaries, Appl. Math. Finan., 13 (2006), 155-190.

[4] S. Kusuoka, M. Ninomiya and S. Ninomiya, Application of the Kusuoka approximation to barrier options, CARF Working Paper, CARF-F-277, The University of Tokyo, (2012), 1-8.

[5] T. Kato, A. Takahashi and T. Yamada, A semigroup expansion for pricing barrier options, Int. J. Stoch. Anal., 8 (2014), $1-15$.

[6] E. Gobet and A. Kohatsu-Higa, Computation of greeks for barrier and lookback options using Malliavin calculus, Electron. Commun. Probab., 8 (2003), 51-62.

[7] T. Funaki and K. Ishitani, Integration by parts formulae for Wiener measures on a path space between two curves, Probab. Theory Relat. Fields., 137 (2007), 289-321. 